\title{
A Kalman Filter Process for Energy Optimization in WSNs
}

\author{
Imad Iala, Imane Dbibih, Ouadoudi Zytoune, Mohammed Rziza, and Driss Aboutajdine
}

\begin{abstract}
Wireless sensor networks (WSNs) consist of a large number of small interconnected devices used to measure physical quantities or to monitor an event in a given area. These devices are called sensor nodes and are characterized by low memory, limited computing resources and they are usually powered by an irreplaceable battery. Therefore, it is essential to design and develop an effective and energy aware protocols allowing these nodes to minimize their energy consumption in order to extend the network lifespan. In this article, a new communication mechanism for IEEE 802.15.4 based WSNs called "Kalman Filter based MAC (KF-MAC) protocol" is proposed. KF-MAC is designed to maximize the efficiency of nodes energy consumption. As a result, the nodes lifespan will be extended as much as possible through Kalman predicting filter. The main KF-MAC purpose is to optimize the sleep interval of nodes between consecutive wake-ups. The sensor node should be awake only if it has to receive or to transmit data without introducing unnecessary overhearing or idle listening cost. In other words, there will be an adaptation between the activation of the transceivers and the node traffic flows. More importantly, KF-MAC uses a postponingbased retransmission mechanism that can efficiently resolve the wireless collisions problem caused by data flow contention.

To validate KF-MAC protocol, we compare KF-MAC performances to some existing energy-efficient MAC protocols such as S-MAC protocol and IEEE 802.15.4 standard. The simulation results show that KF-MAC obtains better performance in terms of energy efficiency, Packet Delivery Ratio (PDR), the whole without affecting negatively the latency.
\end{abstract}

Index Terms-WSN, Mac protocol, IEEE 802.15.4 standard, Energy consumption, Kalman filter.

\section{INTRODUCTION}

$\mathbf{I}$ $\mathrm{N}$ recent years, Zigbee / IEEE 802.15.4 has been one of the most widely used technologies in scientific and industrial applications [1] [2]. Especially, it was designed for Wireless sensor network (WSN). This latter is generally composed of a large number of low-cost devices called sensor nodes. These nodes are scattered in the monitored area [3] and integrate many units (wireless communication unit, computing unit and sensing unit), in addition, they are usually powered by an irreplaceable limited battery, which limits their lifespan and therefore the lifespan of the entire network.

Manuscript received May 23, 2018; revised November 14, 2018. Date of publication January 16, 2019. The associate editor Prof. Joško Radić has been coordinating the review of this manuscript and approved it for publication.

I. Iala, I. Dbibih, M. Rziza and D. Aboutajdine are with the LRIT Associated Unit to the CNRST-URAC N29, Faculty of Sciences, University Mohammed V, B.P.1014 RP, Rabat, Morocco (e-mails: imad.iala01@gmail.com imane.it@gmail.com, mohammed.rziza@gmail.com, aboutaj@hotmail.com).

O. Zytoune is with the ENCG, University Ibn tofail Kenitra, Morocco (email: zytoune@univ-ibntofail.ac.ma).

Digital Object Identifier (DOI): 10.24138/jcomss.v15i1.561
IEEE 802.15.4 standard is mainly addresses the energy lack in WSN. For this reason, most of existing sensors on the market use this technology. The energy waste at nodes comes from many sources (e.g., communication, processing, and sensing). And according to the research work that has been proposed in optimization energy consumption context, the radio interface is typically the most consuming component. Hence, the IEEE 802.15.4 MAC layer uses duty cycling approach for reducing the transceiver energy consumption and thus prolonging the network lifespan as long as possible.

In the IEEE 802.15.4 standard, the coordinator node, periodically, broadcasts beacon frames. Each one contains information about network configuration and data allowing nodes to know when they need to wake up to communicate effectively. The time interval between two beacon frames is called super-frame and which includes two periods, an active and inactive part. Fig. 1 shows the IEEE 802.15.4 super-frame configuration.

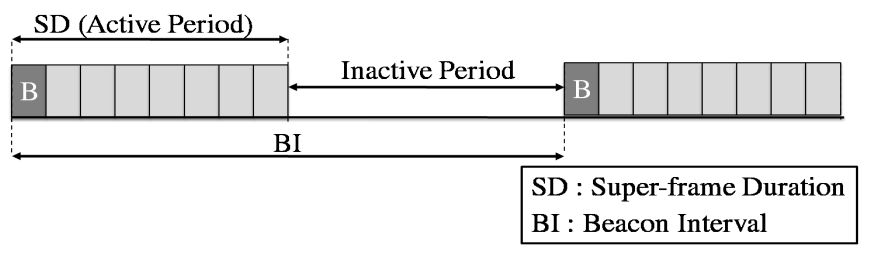

Fig. 1: IEEE 802.15.4 super-frame configuration

The first period is divided into several equal duration time slots; the beacon always occupies the time slot 0 . Normally, the network nodes wake up before time slot 0 and start listening to receive the beacon from coordinator node. After extracting the beacon frame information, the nodes become aware of the current super-frame configuration. If they have no data to transmit or to receive, they go into standby mode until the next super-frame; otherwise they go into sleep mode as soon as their transactions (transmission and / or reception) are finished.

In present paper, we propose a novel energy-efficient protocol to optimize sensor nodes energy consumption and extend the network lifespan. The perfect way to reduce power consumption is to turn $\mathrm{ON}$ the nodes transceiver only if they have to transmit or to receive data. As a result, the sensor nodes wake up less frequently and therefore consume less energy. To this purpose, KF-MAC protocol makes use of Kalman filter. This latter predicts the next state of a system depending on the previous state and the current measurements without using the history of all observations. To KF-MAC, the system corresponds to each node transceiver. It has to 
predict the next state of a transceiver according to the current data receiving moment without using all the history of data receiving moments. In other words, the nodes waking instants will be computed by KF-MAC based only on the last data receiving moment.

The rest of the article is organized as follows: Section II summarizes related works. Section III presents the network model and design principles. Section IV explains KF-MAC process and its features. Simulations results and discussions are presented in Section V. Finally we conclude our paper in Section VI.

\section{RELATED WORKS}

In the MAC layer, most of the energy waste is due to the idle listening. The sensor nodes must keep the transceiver in listening even if they are in standby mode. The IEEE 802.15.4 standard [4] was designed for the Low Rate Wireless Personal Area Networks (LR-WPAN). This standard has many drawbacks in terms of energy consumption, reliability and delay [5]. Therefore, we have to design and develop new protocols to prolong the network lifespan and improve the service quality [6] [7] [8].

A-MAC is proposed by Dutta et al. in [9], this protocol supports broadcast, unicast, pollcast and wake-up services under a unified architecture. SRI-MAC (Synchronous Receiver Initiated) MAC protocol is proposed by Boulfekhar et al. in [10], it is based on the operating of synchronized dutycycling MAC protocol, and which uses an adaptive beacon and RTS/CTS packets for minimizing the idle listening and reducing the duty-cycle. Dong et al. [11] have designed a Prediction-Based Asynchronous MAC protocol called (PBAMAC) for heavy traffic load in WSNs. this protocol applies a wakeup mechanism to save the energy of the sensor nodes. This mechanism allows sensor nodes to reduce communication costs by enabling the sender node to predict the wakeup time of the destination node, without introducing unnecessary overhearing or idle listening cost. In addition, its retransmit process based on the backcast can effectively resolve severe wireless collisions caused by concurrent traffic flows. Tang et al. proposed PW-MAC (Predictive-Wakeup) MAC protocol in [12]. PW-MAC uses a predicting method to estimate the target nodes wake-up time so that a sender needs to wake up before the target receiver. Peng et al. proposed Cy-MAC in [13], it adjusts dynamically the network node's duty-cycling according to the varying traffic conditions by planning the rendezvous schedules between neighbors. Qiu et al. proposed a single-hop broadcast protocol for asynchronous receiver-initiated MAC in WSN in [14] called RWB, this protocol predicts the nodes wake-up time and cuts the long back-to-back broadcast into several unicast packets in the aims of reducing the energy consumption. Tang et al. proposed HKMAC protocol in [15], this protocol provides a low packet delivery latency and high energy conservation using the burst traffic by adjusting receiver's beacon time and scheduling the listening time of the sender nodes during scheduled period. An energy-efficient Multi-Layer MAC (ML-MAC) protocol for WSNs is proposed by JHA et al. in [16]. It is designed with low duty cycle to reduce the energy required to communicate and low number of collisions in cases where two or more nodes try to send data at the same time. Weather Adaptive receiver-initiated MAC protocol (WA-MAC) is proposed by Tian et al. in [17], it adjusts the data transmission process to avoid packets being lost, and it establishes appropriate rendezvous time between receivers and senders to reduce the energy consumption for sensors during data transmission. RAMADAN et al. proposed a Node-power-based MAC protocol with adaptive listening period for WSNs in [18]. For maximizing the energy efficiency, this protocol introduces some message latency to reduce the energy loss duo to idle listening. Zhao et al. proposed a simple collision-avoidance MAC (E-MAC) protocol in [19], it achieve collision-free access. In this protocol each transmitter has to adjust its next transmission time according to which part of its packets suffering from the collision. Renato et al. [20] proposed a MAC (RITMC) initiated by an asynchronous multichannel receiver for the low power WSN. Through this protocol, the authors tries to mitigate the effect of idle listening and the confinement of messages by the use of a recognition mechanism (HA : hello acknowledgment) proposed by the A-MAC protocol, a multichannel mechanism that allows the node to uses its previous knowledge of the neighbor's best channel to communicate with it, and an efficient diagnostic. In order to evaluate the importance of QoS in the MAC protocol for WSN, Subramanian et al. [21] proposed a new MAC QoS protocol called "PRIority in Node (PRIN)", this protocol uses three priority levels to prioritize the traffic, for each of these priority levels, priority queues are defined for different classes of traffic. The highest priority data are served by the node. Sakya et al. [23] suggest a mission-critical MAC protocol called (MC-MAC) which uses an adaptive service cycle approach based on regression. In this protocol, the duty cycle is given by the traffic regression model while taking into account the performance of the S-MAC protocol for packet delivery ratio, throughput, and residual energy. Sundararaj et al. [24] propose an Energy Efficient Dynamic Scheduling Hybrid MAC Protocol named (EDS-MAC). This protocol operates in two stages. In the first stage "cluster formation $\check{T}$, the authors developed a Variable Step Size Firefly Algorithm (VSSFFA) to generate energy-aware clusters by optimal selection of cluster heads. The VSSFFA method allows reducing the cost of locating optimal position for the head nodes in a cluster. While in the second stage "data transmission", the authors aim to reduce the delay, latency, and control overhead by minimizing the size of control and enabling lower duty cycle operation. Swain et al. [25] proposed an energy efficient multichannel MAC protocol called "Advertisement Based Multichannel Distributed MAC Protocol" (Adv-MMAC), this protocol allows nodes to dynamically switch their radio interface between the channels. The proposed protocol consists of two steps, during the first step, the nodes that have data try to contend the advertisement channel to transmit the ADV packet. After receiving the acknowledgment from the receiver, the nodes switch to the available free channel for transmitting the data packet. Khalil et al. [26] proposed an improvement of (RIMAC) protocol called (DURI-MAC). This protocol uses dual channel in order to reduce the number of hidden and exposed 
terminal that minimizes the number of collision, and enhances the system throughput. In addition, it uses a unique CCA time generator that also improves the network throughput. AlSulaifanie et al. [27] proposed an Adaptive Hierarchical MAC protocol named (AH-MAC) with cross-layer optimization for low-rate WSNs. The main objective of this protocol is to combine the strong points of LEACH protocol and the IEEE 802.15.4 standard while compensating for their weaknesses. The predetermined cluster heads are equipped with an energy recovery circuit, while the normal nodes are powered by battery. To extend the operational lifespan of the network, AH-MAC protocol transfers most of the network's activities to cluster heads while reducing the node activity. Iala et al. [28] proposed a new protocol based on IEEE 802.15.4 standard called "Adaptive Duty-cycle (AD-MAC) protocol" for reducing the energy dissipation where the transceiver is waiting potential packets. This protocol uses a new predicting mechanism to estimate the moment when nodes should be awake or asleep according to previous traffic load statistics. AD-MAC protocol allows nodes to adapt the states of their transceiver with network traffic load.

\section{Network MOdel AND DESIGN PRINCIPLES}

In the literature there are many prediction filters, these filters have become essential tools in modern science. They perform state prediction and parameter estimation in several domains, such as robotics, computer vision and network communications, etc. When we study and resolve a problem, we first need its proper mathematical formulas, finding the essential parameters that better describe our system and modeling their behavior over time. Generally, predictive filters estimate the optimal state of a system. First, they use the mathematical model of the system dynamics to propagate the state's values and uncertainties. Then, they combine this preliminary estimate and the best that can be used from the observation.

There are several predictive filters, each appropriate for a different type of uncertainty representation and dynamic modeling. In this paper we focus our study on Kalman filter; this filter represents uncertainties as Gaussian random variables, fully described by a mean and a covariance matrix, and models the system with linear dynamics and observations. And the gaussians are preserved under linear transformations. The strength of Kalman filter is its ability to predict parameters and rectify errors, not only of sensors, but also of the model itself. Indeed, to apply a Kalman filter, we must first model the system for which we want to estimate the parameters linearly. In a classical estimation methods (for example, the Least squares approximation method), a simple error in the system modeling inevitably leads to an error in the estimation. The stoutness of Kalman filter is to integrate an imprecision term in the model itself, which allows it to give correct estimates despite modeling errors. It is for all these reasons that we have chosen Kalman filter regardless of its simplicity of implementation.

We consider that the coordinator node has an unlimited source of energy and periodically broadcasts beacons frames. Thereby, we should not worry about its energy consumption.
The other sensor nodes have their own configuration of the Super-frame Duration (SD) period, which is update every super-frame according to Kalman filter process. In Fig. 3 we explain how the nodes SD configuration is updated.

At the beginning, all time slots of the SD are set to the active state. Keeping the first time slot in the active state, allows sensor nodes to wake up and receive the beacon frame in order to synchronize. An illustrative example is depicted in Fig. 2. In this example, the network is composed by three sensor nodes and a coordinator node. Note that, the analysis that follows is general and can be applied to any number of sensor nodes. In this scenario, the coordinator node is always in active mode, and periodically broadcasts beacons frames. Thus, each node stores the moments when it has received data in the previous super-frame. The proposed network architecture is as follow:

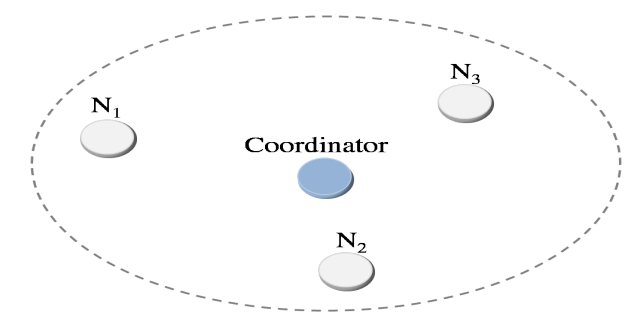

Fig. 2: Topology of proposed example

Initially, all nodes set $\mathrm{ON}$ all time slots of active part of the first Super-frame. Assuming that the node $N_{2}$ received a data packet from the node $N_{1}$ at the time $T_{1}$, and another data packet from $N_{3}$ at the instant $T_{2}$. In this case, $N_{2}$ stores these two moments. Then, it applies the Kalman filter to estimate the next moments when it should wake up to probably receive data. The estimated moments are adjusted to be in the interval $\left[0,2^{S O} \times 15.36 \mathrm{~ms}\right]$ where SO (Super-frame Order) is a parameter used to fix the active duration (SD) of a superframe.

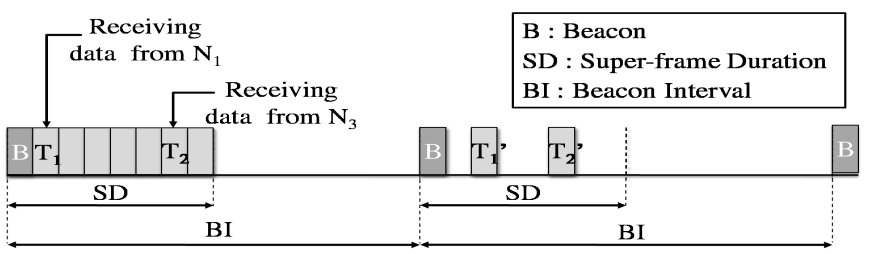

Fig. 3: Super-frame configuration of Node $N_{2}$

In Fig. 3 we have $T_{1}^{\prime}$ and $T_{2}^{\prime}$ are the estimated moments by $N_{2}$ using Kalman filter, and which represent the times when the next data reception may occur. The next superframe configuration of $N_{2}$ will be changed, only the first time slot, surely, have to be active, however, the others time slots state is based on Kalman filter computation. According to this scenario, $N_{2}$ should be awake during $T_{1}^{\prime}$ and $T_{2}^{\prime}$ to probably receive data from its neighbors.

In Fig. 4, we suppose that the node $N_{3}$ wants to transmit data at instant $T_{3}$ to $N_{2}$. Unfortunately, this latter is currently in sleep mode because its Kalman filter did not predict this event. Normally, The IEEE 802.15.4 standard considers a node unreachable if this one can't receive data after four transmission attempts. Thus, the sender node deletes this 
packet and aborts this communication. As a result, we lose data that can be sensitive. Although in KF-MAC, node $N_{2}$ can be just asleep because of Kalman filter computation. To resolve this problem, we developed a novel postponing-based retransmission mechanism, in which, we force the sender node $\left(N_{3}\right)$ to inform the coordinator node (when the receiver node $\left(N_{2}\right)$ is still unreachable after the third transmission attempt) via a new control packet called PDI (Postponed Data Information). The PDI packet is constructed as mentioned in Table. I. PDI packet contains the receiver node ID $\left(N_{2}\right)$ and the instant of the first transmission attempt when $N_{3}$ wanted to send data to $N_{2}$. Concerning the fourth and the last attempt, the node $N_{3}$ postpones it until the next super-frame.

TABLE I: POSTPONEd DATA INFORMATION PACKET

\begin{tabular}{|c|c|c|c|c|}
\hline Octets : 2 & 1 & 2 & 2 & 2 \\
\hline \hline $\begin{array}{c}\text { Frame } \\
\text { Control }\end{array}$ & $\begin{array}{c}\text { Sequence } \\
\text { Number }\end{array}$ & $\begin{array}{c}\text { Concerned } \\
\text { Node ID }\end{array}$ & $\begin{array}{c}\text { Instant of the } \\
\text { First attempt }\end{array}$ & $\begin{array}{c}\text { FCS (Frame } \\
\text { Chek Sequence) }\end{array}$ \\
\hline \multicolumn{2}{|c|}{ MHR } \\
(MAC Header)
\end{tabular}

The field order of PDI packet must respect the general MAC frame order [4]. The MHR of PDI packet contains only the frame control field and the sequence number field. In the Frame Control field, the frame type subfield contains a value different to these used by the MAC command, acknowledgment, the beacon and data. We use the value 7 to indicate a PDI packet. The Sequence Number field contains the same value of the data frame sequence number. The MAC payload for a PDI packet includes two fields: the first field contains the concerned node ID, while the second field contains the instant when the first transmission attempt occurred. The MFR is composed of a 16 bit FCS.

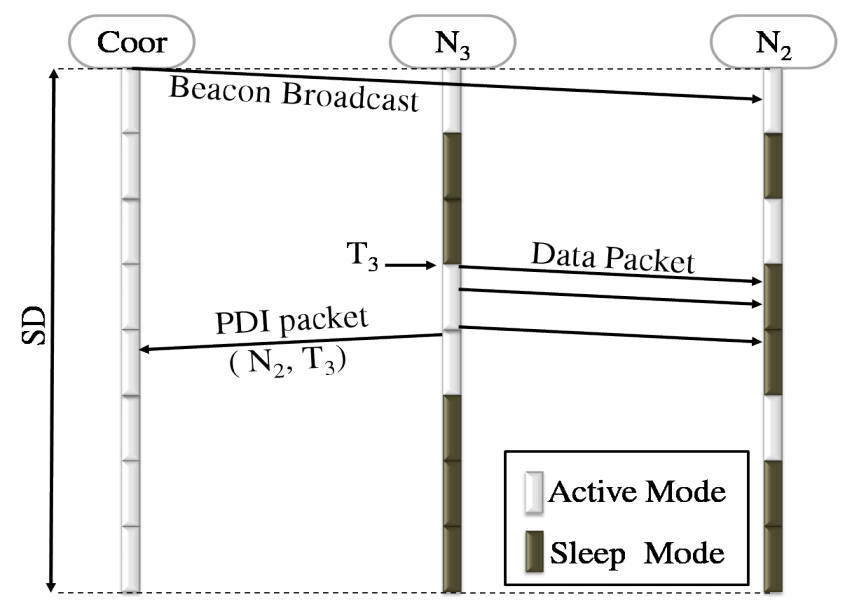

Fig. 4: Exchange message scenario

When the coordinator node receives the PDI packet, it extracts the both information $\left(N_{2}\right.$ ID and the first transmission attempt instant) and inserts them into new fields of the next beacon frame. All sensor nodes are listening the beacon frame, in this example, only the node $N_{2}$ which has its ID equal to the node's ID inserted in the extended field of the beacon frame is concerned to extract and store the instant $T_{3}$ in order to include it in the next Kalman filter computation.

\section{KF-MAC PROCESS}

In this paper, we propose a novel Energy Efficient Mac protocol to dynamically control the wake up moments of sensor nodes where they have probably data to receive. For this purpose, KF-MAC protocol consists in adapting the active state of the sensor nodes transceivers to the traffic flows by using the Kalman filter.

\section{A. The Process Model}

To estimate the moments when the nodes have to wake up, KF-MAC takes the data receiving moments in the previous super-frame as measurements. However, in real life applications of WSNs, there is always a noise factor called the Gaussian noise that can disturb our measurements. For this, our process is described by the following linear difference equation:

$$
x_{k+1}=A \times x_{k}+V_{k}(\text { Estimated_state })
$$

Where $x_{k+1}$ is the process state vector at step $k+1, A$ is the matrix of state transition from step $k$ to step $k+1, x_{k}$ is the vector of process state at step $k$ and $V_{k}$ is a vector assumed to be a white sequence with known covariance structure (the state noise). The observation/measurement of the studied process is assumed to occur at a discrete time in accordance with:

$$
z_{k}=B \times x_{k}+W_{k}(\text { Covariance_estimation })
$$

$z_{k}$ is the measured value of the system and it relates to the computed value $x_{k}$. In a perfect case, $z_{k}=x_{k}, B$ is an identity matrix, it relates the state to the measurement $z_{k}$, and $W_{k}$ is the measurement error vector (the observation noise). In our case $z_{k}$ corresponds to the moment where the node has received a data packet.

While functioning, the state of our system is always the same, so $A=1$. Our noise measurement factor is directly considered in the measured value of the system, so $B=1$. (Notice that we dropped the subscript $k$ in several places because the respective parameters remain constant in our model). Thus, our equations (1) and (2) become as follows:

$$
\begin{gathered}
x_{k+1}=x_{k}+V_{k} \\
z_{K}=x_{k}+W_{k}
\end{gathered}
$$

The Kalman filter operates in two distinct stages [29]: Predict step witch we use the estimated state of the previous active time to produce an estimate of the current state. Initially, to calculate the optimal estimate, we first calculate the weight ' $K_{k}$ ':

$$
K_{k}=\frac{P_{k}^{\prime}}{P_{k}^{\prime}+R}
$$

Where $R$ presents the measure variance that is within the range of \pm time slot of the true value of data reception moment, and $P_{k}^{\prime}$ is the Guess Variance. At the beginning, we need to choose an initial value for $P_{k}$, i.e. $P_{k}^{\prime}$. If our initial 
estimated state $\hat{x}_{k}^{\prime}=0$, we would let $P_{k}^{\prime}=0$. However given the uncertainty in our initial estimate $\hat{x}_{k}^{\prime}$, choosing $P_{k}^{\prime}=0$ would take the filter to always believe that $\hat{x}_{k}=0$. As it turns out, the alternative choice is critical. We have to choose almost any $P_{k}^{\prime} \neq 0$ and the filter would eventually converge. In our case we will start our filter with $P_{k}^{\prime}=1$. That means, we trust the measurement more than we trust our guesses.

$K>1$ means the measurement is more trustworthy.

$K>0$ means our guess is more trustworthy.

From experience, we know that the true values of the data reception moment have a standard normal probability distribution, we will start our filter with the guess that the moment is 0 . In other words, before starting we let $\hat{x}_{k}^{\prime}=0$. And we calculate the estimate:

$$
\hat{x}_{k}=\hat{x}_{k}^{\prime}+K \times\left(z_{K}-\hat{x}_{k}^{\prime}\right)
$$

Then, we need to calculate the estimate variance $P_{k}$.

$$
P_{k}=\left(1-K_{k}\right) \times P_{k}^{\prime}
$$

In Kalman filter, we predict, update and repeat the process as required. In our case, this process will be repeated for each measurement (data reception moments). Our moments update equations are:

$$
\begin{aligned}
& P_{k+1}^{\prime}=P_{k} \\
& \hat{x}_{k+1}^{\prime}=\hat{x}_{k}
\end{aligned}
$$

\section{Performance Evaluation}

\section{A. Simulation Parameters}

In this section, we validate the KF-MAC protocol analytical model by evaluating its performances using NS-2 simulator. The results are compared with those of IEEE 802.15.4 standard and S-MAC protocol. The evaluation focuses on how the sensor nodes save power without affecting the PDR and latency.

In this simulation, we consider a scenario containing 99 sensor nodes connected to a coordinator node (100 sensor nodes in total). The coordinator node has an unlimited source of energy. All this sensor nodes use the KF-MAC protocol and they are randomly deployed in the monitored area. To validate KF-MAC protocol performances, we evaluate end-toend delay (latency), delivered packet rate (PDR), and energy consumption. For this goal, first, we use Constant Bit Rate (CBR) traffic, second, we use exponential traffic. The results of the simulation are carried out with parameters presented in Table II.

\section{B. Simulation Results}

For simulations, we are interested by the Beacon-Enabled operation mode of IEEE 802.15.4 standard. We use two scenario where the sensor nodes are randomly deployed. First, we consider a collected data scenario of environmental monitoring with exponential packet arrivals time characterized by an average rate of $\lambda \mathrm{PCKT} / \mathrm{s}$. Second, we consider a periodically collected data scenario.
TABLE II: Parameters Values of Simulation

\begin{tabular}{|l|l|}
\hline Parameters & Values \\
\hline \hline Number of nodes & 100 \\
\hline Propagation mode & Two-Ray-Ground \\
\hline Routing protocol & AODV \\
\hline Type of topology & Mesh \\
\hline Topology dimension & $300 \mathrm{x} 300 \mathrm{~m}^{2}$ \\
\hline Data packet size & $100 \mathrm{~B}$ \\
\hline Radio power in idle and listen mode & $14.4(\mathrm{~mW})$ \\
\hline Radio power in transmitting mode & $36.0(\mathrm{~mW})$ \\
\hline Radio power in sleep mode & $0.015(\mathrm{~mW})$ \\
\hline Simulation time & $1000(\mathrm{~s})$ \\
\hline
\end{tabular}

1) CBR Traffics: In first simulations stage, we fixed the number of connection (number of communications) at 10 , and we varied the packets transmission interval from $0.5 \mathrm{~s}$ to 2.5. In the second stage, we fixed the packet transmission interval at $1.5 \mathrm{~s}$, and we varied the number of sender nodes from 5 to 25 . This helps us to evaluate the impact of the communication load and the impact of the traffic density on the various performances metrics. All results are the average of 50 simulations.
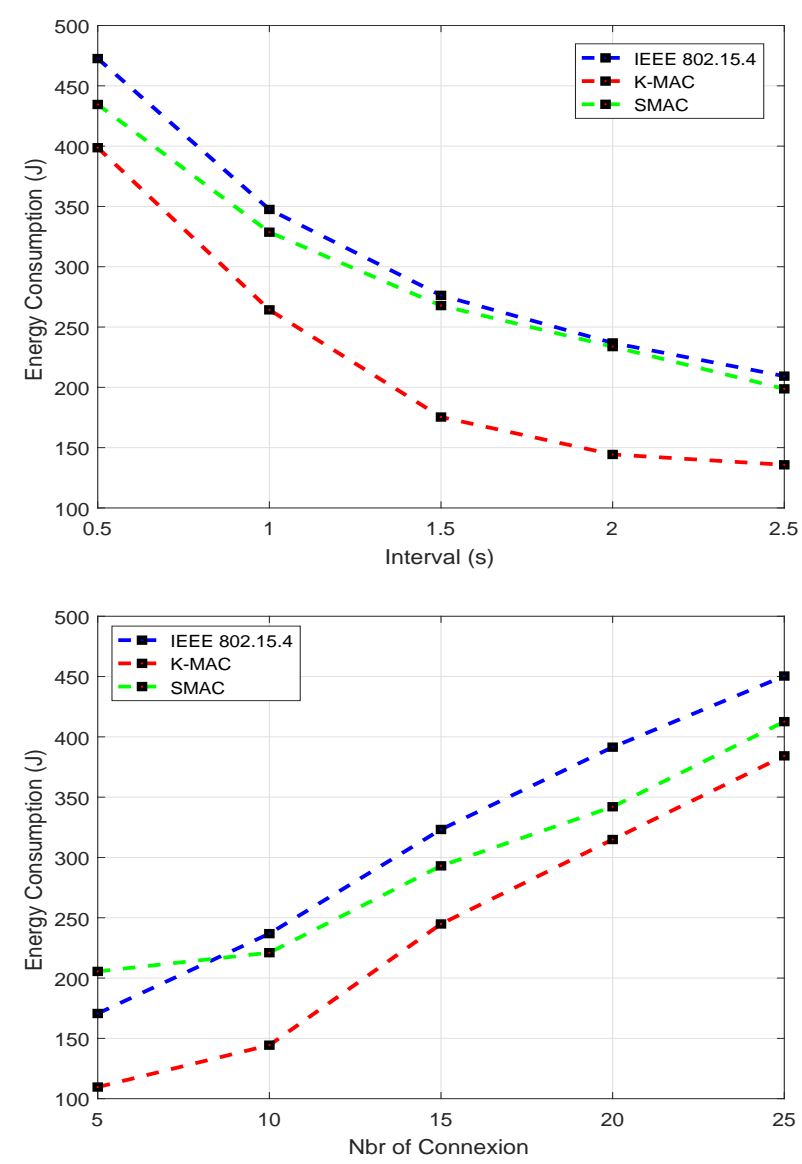

Fig. 5: Energy consumption results

As a first comparison of performance metrics, we measured the average of energy. The energy average consumed during the simulation was obtained by the sum of the energy consumed at the end of the simulation of each node divided by the total number of nodes. The energy consumption increases 
proportionally with the number of packets. Fig. 5 shows that KF-MAC protocol consumes always less energy than those of IEEE 802.15.4 standard and S-MAC, whether in the case of low traffic or in the case of a high density of traffic. This is caused by the more asleep of sensor nodes, thanks to the prediction mechanism which wakes up the nodes only if an event (receiving data) will probably occur, the sensor nodes will not waste energy due to idle listening or overhearing, which allows them to stay longer alive. And consequently, the entire network lifespan will be extended.
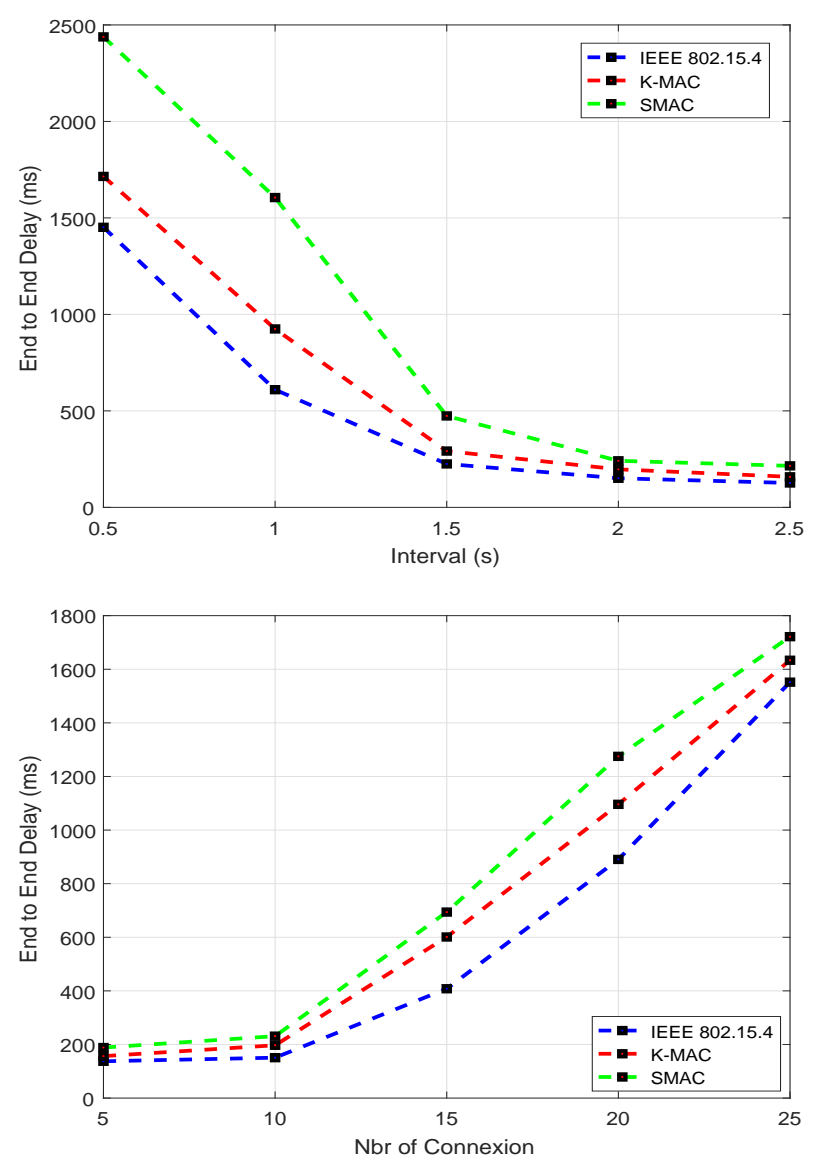

Fig. 6: The average latency results

End to End delay measures the average time cost between the receive and the send moment of data packets. It represent the stability and reliability of the WSN. More the latency is reduced, the protocol is found to be more robust. Fig. 6 shows that End to End delay of KF-MAC knows a small increase compared to those of the IEEE 802.15.4 standard and S-MAC protocol. The reason behind the increased delay, is the fact of always postponing the last data transmission attempt until the next super-frame. In addition, when there are more contending senders nodes, the probability to have more collisions increase, the backoffs process can degrade the QoS of MAC protocols. When the collisions happen, a sensor node must wait for backoff time before to retransmit data packets. Furthermore, persistent collision causes to the retransmission of data packets multiple times, which results in a larger End to End delay.
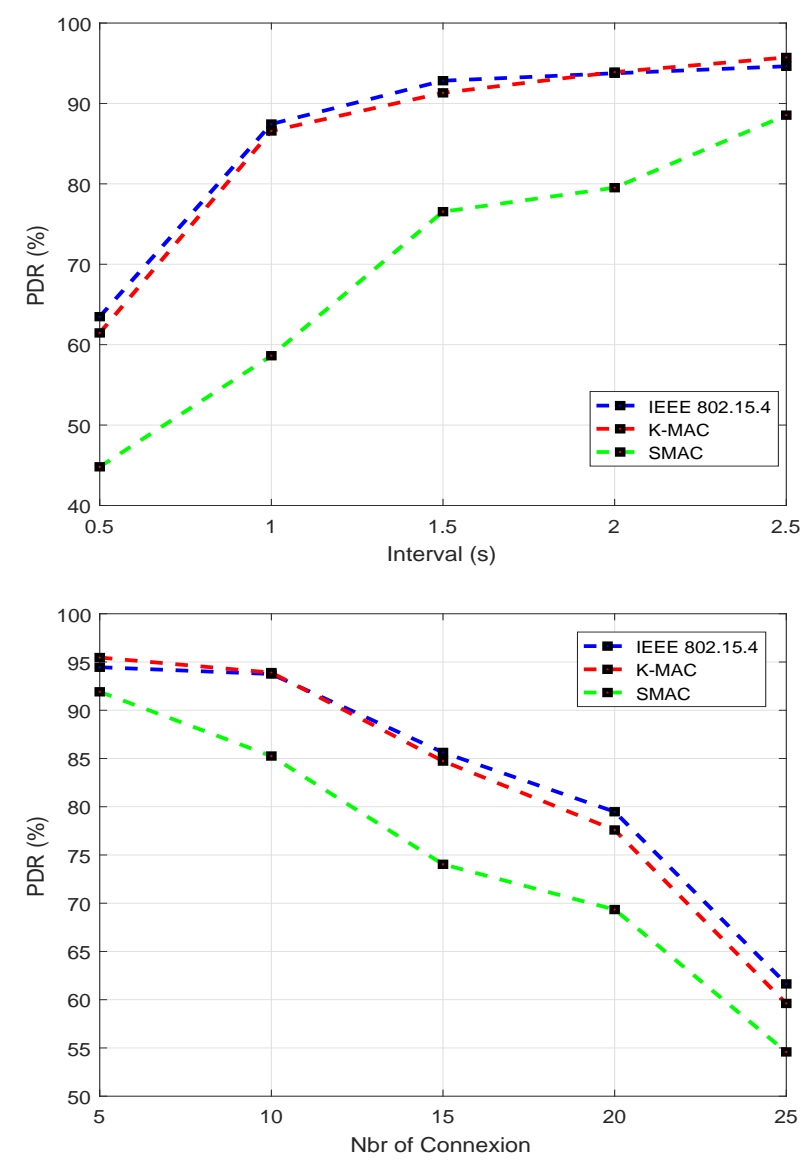

Fig. 7: Packet delivery ratio results

In Fig. 7, PDR decreases in two cases, either in the case when the number of communication in the network increase. Or in the case when the data packet transmission interval decreases. This is due to the growth of the probability of having packet loss due to collisions. The highlight of KFMAC protocol is keeping the same rate of delivered packets as the IEEE 802.15.4 standard in the all evaluation cases, even if there is a high probability of having the destinates nodes in sleeping mode because of prediction mecanism. KFMAC protocol is a efficent solution that allows reserving the energy consumption of the sensor nodes without increasing the number of lost packets.

2) Exponential Traffics: In this part of simulations, we assume that the occurred events follow the exponential distribution. First, We varied the average time between the events from 10 to $210 \mathrm{~ms}$. Second, we fixed the average time between events at $210 \mathrm{~ms}$, and we varied the number of connections (number of communication) from 5 to 25 . This help us to evaluate the impact of the traffic density and the impact of communications load on the various performance metrics of KF-MAC protocol. Over 100,000 events were produced in each simulation case.

Prolonging the network lifespan is the most major challenge to resolve in WSNs, though, It is found that when the generation of data packets increases the energy consumption of sensor nodes also increases. According to Fig. 8, it is 

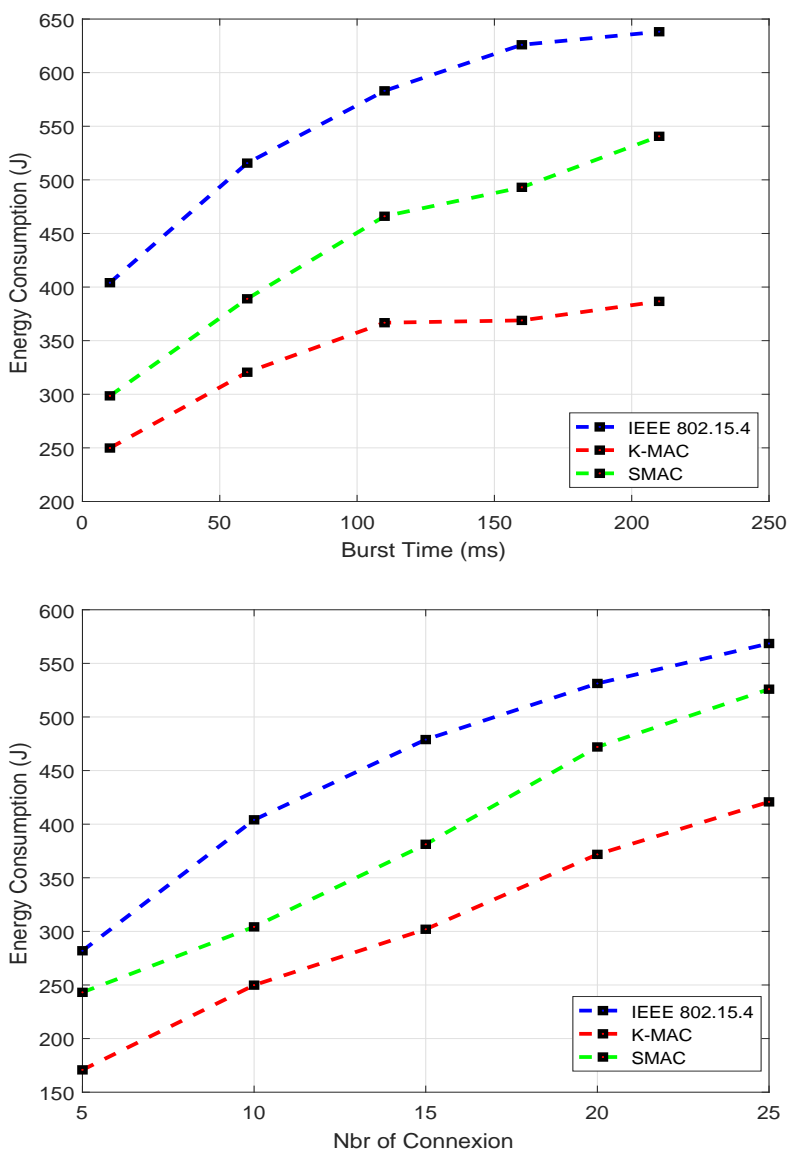

Fig. 8: Energy consumption results

clear that KF-MAC protocol is a better solution to reduce energy consumption of sensor nodes. KF-MAC protocol is widely powerful within the meaning of the reduction of energy consumption compared to IEEE 802.15.4 standard and S-MAC protocol. Whether in the case of low communications load, or in the case of a heavy traffic. This is thanks to Kalman filter computation that estimates the times when sensor nodes must be active to receive data packets.

Concerning the latency in Fig. 9, we note that KF-MAC protocol suffers from a delay compared to IEEE 802.15.4 standard. the End to End delay obtained by KF-MAC protocol knows a small increase. This difference does not exceed 150 $\mathrm{ms}$ in the worst case.

Fig. 10 proves that even in the case of increasing the number of source nodes or in the case of heavy traffic KF-MAC protocol keeps the same PDR than the IEEE 802.15.4 standard. $\mathrm{KF}-\mathrm{MAC}$ protocolis a real solution to save energy nodes in the beacon mode without increasing the number of lost packets and without having a critical latency.

\section{CONCLUSION AND Future WORK}

In this work, an effective and energy aware protocol (KFMAC Protocol) has been presented. KF-MAC is a good solution for reducing the power consumption of WSN thanks to its process that wakes nodes up only if they should transmit or receive data packets. The effectiveness of this solution has
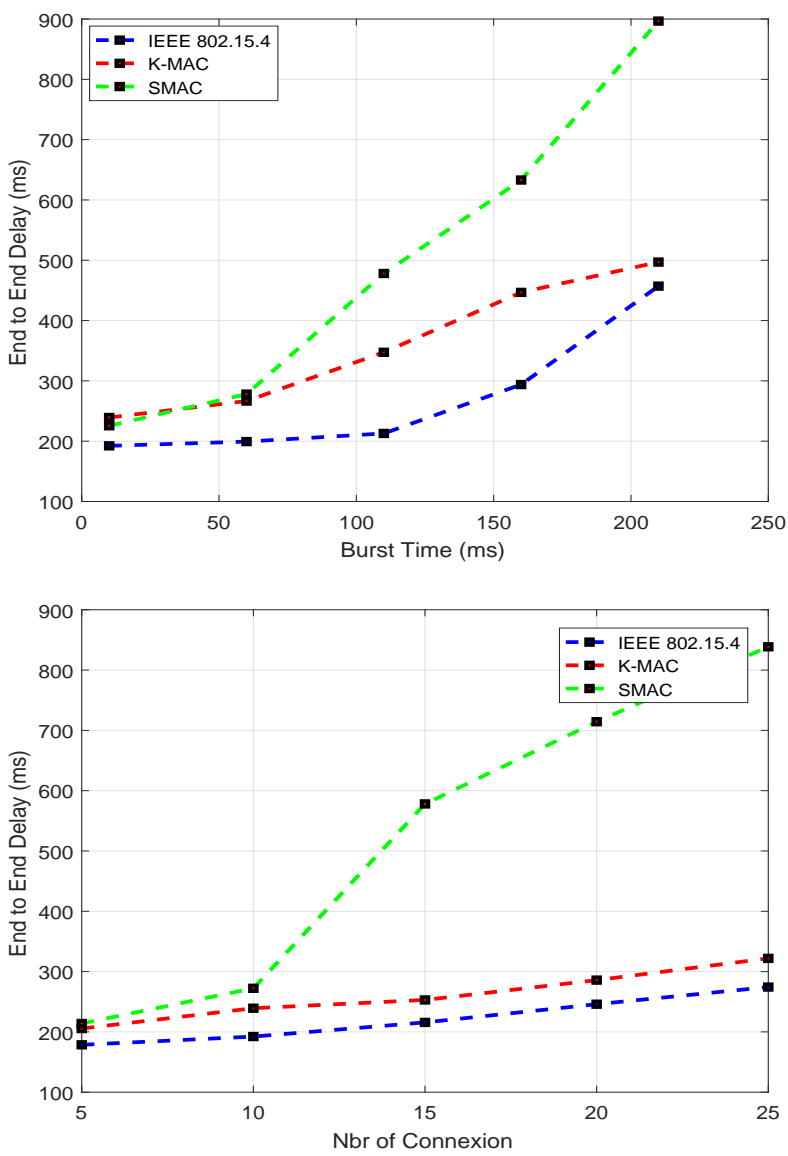

Fig. 9: The average latency results

been proved by the obtained results in several scenarios, either in low and high traffic load scenarios, or in low and high communications load scenarios. Simulations confirmed that through KF-MAC protocol, we get to optimize the energy consumption and the packet delivery ratio without influencing negatively message latency. We believe that the concept underlying of KF-MAC can be adopted in different topologies, so, in our future work we intend evaluating this protocol in different application environments.

\section{REFERENCES}

[1] P. Baronti, P. Pillai, V. W. C. Chook, S. Chessa, A. Gotta, and Y. F. $\mathrm{Hu}$, "Wireless sensor networks: A survey on the state of the art and the 802.15.4 and ZigBee standards," Computer Communications, vol. 30, no. 7, pp. 1655Ú1695, May 2007, DOI: 10.1016/j.comcom.2006.12.020.

[2] K. Sohraby, D. Minoli, and T. Znati, "Wireless Sensor Networks." John Wiley \& Sons, Inc., 30-Mar-2007, DOI: 10.1002/047011276X.

[3] J. Yick, B. Mukherjee, and D. Ghosal, "Wireless sensor network survey," Computer Networks, vol. 52, no. 12, pp. 2292Û2330, Aug. 2008, DOI: 10.1016/j.comnet.2008.04.002.

[4] LAN/MAN Standards Committee. "Part 15.4: wireless medium access control (MAC) and physical layer (PHY) specifications for low-rate wireless personal area networks (LR-WPANs)." IEEE Computer Society (2003).

[5] G. Anastasi, M. Conti, and M. Di Francesco, "The MAC unreliability problem in IEEE 802.15.4 wireless sensor networks," in Proceedings of the 12th ACM international conference on Modeling, analysis and simulation of wireless and mobile systems - MSWiM '09, 2009, DOI: $10.1145 / 1641804.1641839$. 

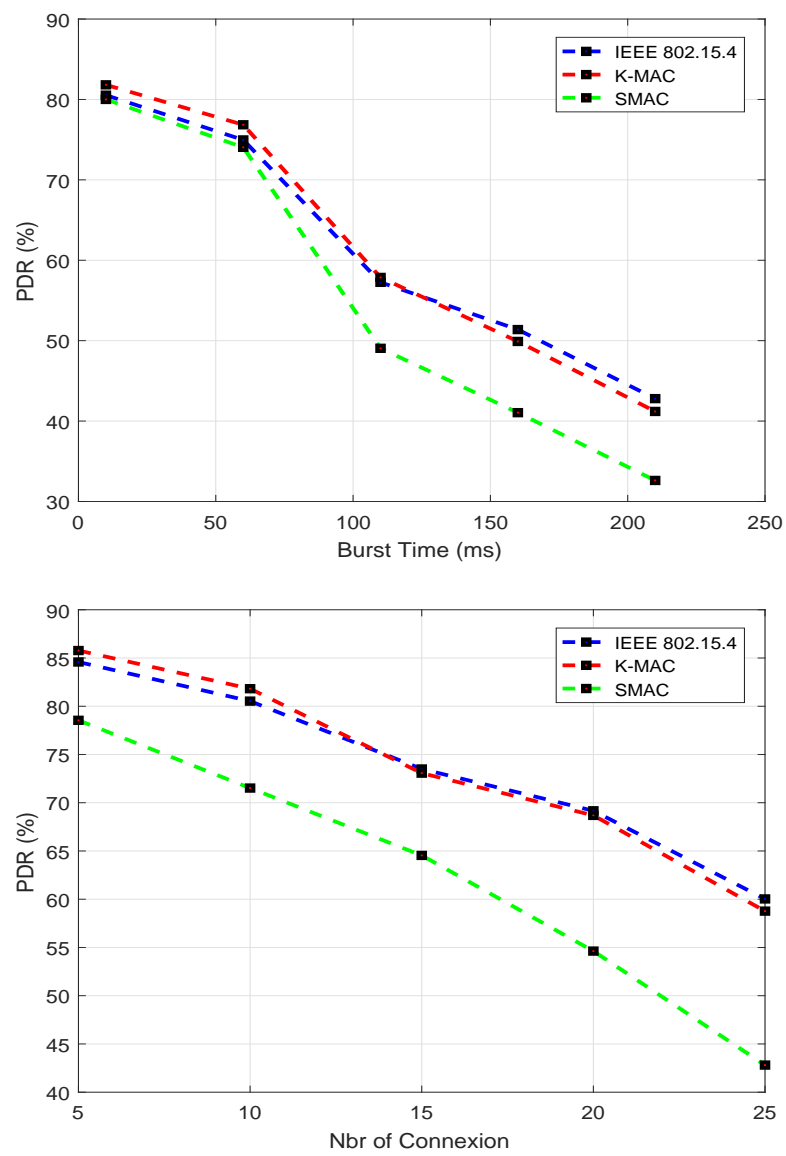

Fig. 10: Packet delivery ratio results

[6] W. R. Heinzelman, A. Chandrakasan, and H. Balakrishnan, "Energyefficient communication protocol for wireless microsensor networks," in Proceedings of the 33rd Annual Hawaii International Conference on System Sciences, DOI: 10.1109/HICSS.2000.926982.

[7] F. Shu, "Performance evaluation of the IEEE 802.15.4 CSMA-CA protocol with QoS differentiation," in 2008 International Conference on Intelligent Sensors, Sensor Networks and Information Processing, 2008, DOI: $10.1109 /$ ISSNIP.2008.4762034.

[8] A. Mohameden, L. Boukhatem, and G. Pujolle, "Class Aware Duty Cycle (CADC) to improve QoS of IEEE 802.15.4 networks," in 2009 2nd IFIP Wireless Days (WD), 2009, DOI: 10.1109/WD.2009.5449697.

[9] P. Dutta, S. Dawson-Haggerty, Y. Chen, C.-J. M. Liang, and A. Terzis, "A-MAC," ACM Transactions on Sensor Networks, vol. 8, no. 4, pp. 1Û29, Sep. 2012, DOI: 10.1145/2240116.2240119.

[10] S. Boulfekhar and M. Benmohammed, "Synchronous receiver initiated MAC protocol for long-lived sensor networks," Computers \& Electrical Engineering, vol. 40, no. 2, pp. 504Û́516, Feb. 2014, DOI: 10.1016/j.compeleceng.2013.07.011.

[11] C. Dong and F. Yu, "A prediction-based asynchronous MAC protocol for heavy traffic load in wireless sensor networks," AEU - International Journal of Electronics and Communications, vol. 82, pp. 241Ú250, Dec. 2017, DOI: 10.1016/j.aeue.2017.09.003.

[12] L. Tang, Y. Sun, O. Gurewitz, and D. B. Johnson, "PW-MAC: An energy-efficient predictive-wakeup MAC protocol for wireless sensor networks," in 2011 Proceedings IEEE INFOCOM, 2011, DOI: 10.1109/INFCOM.2011.5934913.

[13] Y. Peng, Z. Li, D. Qiao, W. Zhang, "Delay-bounded mac with minimal idle listening for sensor networks," in: INFOCOM, 2011 Proceedings IEEE, IEEE, 2011, pp. 1314Ü1322.

[14] Y. Qiu, S. Li, D. Yang, and Z. Li, "RWB: An Efficient ReceiverInitiated Single-Hop Broadcast Protocol for Asynchronous MAC in Wireless Sensor Networks," in Recent Advances in Computer Science and Information Engineering, Springer Berlin Heidelberg, 2012, pp. 261Ű266, DOI: $10.1007 / 978-3-642-25769-8 \_38$.
[15] H. Tang, C. Sun, Y. Liu, and B. Fan, "Low-latency asynchronous dutycycle MAC protocol for burst traffic in wireless sensor networks," in 2013 9th International Wireless Communications and Mobile Computing Conference (IWCMC), 2013, DOI: 10.1109/IWCMC.2013.6583594.

[16] M. K. Jha, A. K. Pandey, D. Pal, and A. Mohan, "An energy-efficient multi-layer MAC (ML-MAC) protocol for wireless sensor networks," AEU - International Journal of Electronics and Communications, vol. 65, no. 3, pp. 209Ú216, Mar. 2011, DOI: 10.1016/j.aeue.2010.03.006.

[17] J. Tian, Y. Wang, X. Liang, G. Wang, and Y. Zhang, "WA-MAC: A weather adaptive MAC protocol in survivability-heterogeneous wireless sensor networks," Ad Hoc Networks, vol. 67, pp. 40Û́52, Dec. 2017, DOI: 10.1016/j.adhoc.2017.10.005.

[18] K. F. Ramadan, M. I. Dessouky, M. Abd-Elnaby, and F. E. Abd El-Samie, "Node-power-based MAC protocol with adaptive listening period for wireless sensor networks," AEU - International Journal of Electronics and Communications, vol. 84, pp. 46Ú56, Feb. 2018, DOI: 10.1016/j.aeue.2017.10.034

[19] H. Zhao, J. Wei, N. I. Sarkar, and S. Huang, "E-MAC: An evolutionary solution for collision avoidance in wireless ad hoc networks," Journal of Network and Computer Applications, vol. 65, pp. 1Ü11, Apr. 2016, DOI: 10.1016/j.jnca.2016.02.010.

[20] R. F. Fernandes, M. B. de Almeida, and D. Brandão, "An Energy Efficient Receiver-Initiated MAC Protocol for Low-Power WSN," Wireless Personal Communications, vol. 100, no. 4, pp. 1517Ú1536, May 2018, DOI: $10.1007 / \mathrm{s} 11277-018-5651-3$.

[21] A. K. Subramanian and I. Paramasivam, "PRIN: A Priority-Based Energy Efficient MAC Protocol for Wireless Sensor Networks Varying the Sample Inter-Arrival Time," Wireless Personal Communications, vol. 92, no. 3, pp. 863Ü881, Aug. 2016, DOI: 10.1007/s11277-016-3581-5.

[22] Sinha, Ashmi, and Atul Kumar Pandey. "An energy-efficient MAC protocol for virtual MIMO communications in WSNs."Wireless Communications, Signal Processing and Networking (WiSPNET), 2017 International Conference on. IEEE, 2017.

[23] Sakya, Gayatri, and Vidushi Sharma. "MAC Protocol with Regression based Dynamic Duty Cycle Feature for Mission Critical Applications in WSN." INTERNATIONAL JOURNAL OF ADVANCED COMPUTER SCIENCE AND APPLICATIONS 8.6 (2017): 198-206.

[24] V. Sundararaj, S. Muthukumar, and R. S. Kumar, "An optimal cluster formation based energy efficient dynamic scheduling hybrid MAC protocol for heavy traffic load in wireless sensor networks," Computers \& Security, vol. 77, pp. 277Ú288, Aug. 2018, DOI: 10.1016/j.cose.2018.04.009.

[25] R. R. Swain, S. Mishra, T. K. Samal, and M. R. Kabat, "An Energy Efficient Advertisement Based Multichannel Distributed MAC Protocol for Wireless Sensor Networks (Adv-MMAC)," Wireless Personal Communications, vol. 95 , no. 2 , pp. 655Ü682, Oct. 2016, DOI: 10.1007/s11277016-3791-x.

[26] M. I. Khalil, M. A. Hossain, and I. Ahmed, "DURI-MAC: A dual channel receiver initiated MAC protocol for wireless sensor network (WSN)," in 2017 International Conference on Electrical, Computer and Communication Engineering (ECCE), 2017, DOI: 10.1109/ECACE.2017.7912971.

[27] A. I. Al-Sulaifanie, S. Biswas, and B. Khorsheed Al-Sulaifanie, "AHMAC: Adaptive Hierarchical MAC Protocol for Low-Rate Wireless Sensor Network Applications," Journal of Sensors, vol. 2017, pp. 1Ú15, 2017, DOI: $10.1155 / 2017 / 8105954$.

[28] I. Iala, I. Dbibih, and O. Zytoune, "Adaptive Duty-Cycle Scheme Based on a New Prediction Mechanism for Energy Optimization over IEEE 802.15.4 Wireless Network," International Journal of Intelligent Engineering and Systems, vol. 11, no. 5, pp. 105Û113, Oct. 2018, DOI: 10.22266/ijies2018.1031.10.

[29] R. E. Kalman, "A New Approach to Linear Filtering and Prediction Problems," Journal of Basic Engineering, vol. 82, no. 1, p. 35, 1960, DOI: $10.1115 / 1.3662552$. 


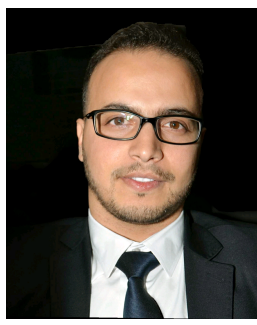

Imad Iala received a License degree in Network and Telecommunications from science Faculty, Rabat (University Mohammed V). In 2010. In 2012, $\mathrm{He}$ received her Master's degree (with honors) in Computer Science and Telecommunications from science Faculty, Rabat (University Mohammed V). Currently, $\mathrm{He}$ is a Ph.D student in the Physical department at the Laboratory for Research in Informatic and Telecommunications (LRIT) in the Faculty of Sciences of Rabat, University Mohammed V Rabat, Maroc. His research interests include the areas of wireless sensor networks, and the optimization of WSN protocols.

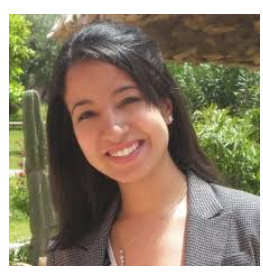

Imane Dbibih received a License degree in Mathematics and Computing from science Faculty, Eljadida (Chouaib Doukkali University) in 2009. In 2011 she received her MasterŠs degree (with honors) in Computer Science and Telecommunications from science Faculty, Rabat (University Mohammed V). She received her Ph.D. in 2016 from the University Mohammed V, Rabat, Morocco. She joined the polydisciplinary faculty of ouarzazat, Ibn Zohr University, AGADIR, Morocco, as an Assistant Professor in November 2016; she is a permanent member of Laboratory of Engineering Sciences and Energy. Her research interests include the areas of wireless sensor networks, and the optimization of WSN protocols

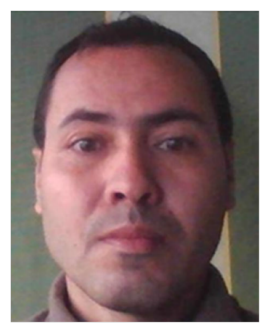

Ouadoudi Zytoune received his Ph.D. in 2010 from the University Mohammed V, Rabat, Morocco. $\mathrm{He}$ joined Ibn Tofail University, Morocco, as an Assistant Professor in November 2010; he is member of ESIT team at Systems Engineering Lab, Associate Researcher at the Laboratory for Research in Informatic and Telecommunications (LRIT) in the Faculty of Sciences in Rabat and LaRIT. His current research interests include Wireless Communications, QoS in Wireless communication and Wireless Sensor Networks.

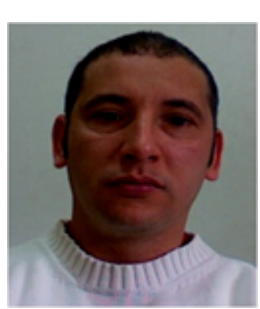

Mohammed Rziza Received the national Doctorate in engineering sciences, Image processing specialty, from the Faculty of Science of the Mohammed V University, Rabat, Morocco, in 2002. He joined the Faculty of Science, Rabat, Morocco, in 2003, as an assistant professor. Since 1997, he is member of the GSCM group. He is also a permanent member of Laboratory for Research in Informatic and Telecommunications (LRIT). His research interests include image processing, pattern recognition, stereovision, and Wireless Communications.

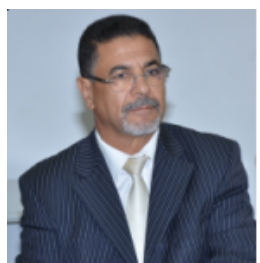

Driss Aboutajdine received the Doctorat de 3' Cycle and the Doctorat d'Etat-es-Sciences degrees in signal processing from the Mohammed V University, Rabat, Morocco, in 1980 and 1985, respectively. He joined Mohammed V University, Rabat, Morocco, in 1978, first as an assistant professor, then as an associate professor in 1985, and full Professor at 1990. From 2013 to 2017 he was the president of the CNRST Morocco. Over 30 years, he developed research activities covering various topics of signal and image processing, wireless communication and pattern recognition which allow him to publish over 300 journal papers and conference communications. 